\title{
Schizophrenia and Suicide: Treatment Optimization
}

\author{
Gaurava Agarwal, MD* \\ Megan Pirigyi, MD \\ Herbert Meltzer, MD
}

\author{
Address \\ *Feinberg School of Medicine, Northwestern University, 446 E Ontario, \\ Chicago, IL 60611, USA \\ Email: gagarwal@nmff.org
}

Published online: 9 March 2014

(C) Springer International Publishing AG 2014

Keywords Suicide - Schizophrenia - Schizoaffective disorder - Nonadherence, Long-acting injectable •

Substance abuse - Clozapine - Side effects - Risk factors, Depression, Antidepressants, Citalopram, Naltrexone

\section{Opinion statement}

In the aftermath of a serious suicide attempt, $25-50 \%$ of patients with schizophrenia or schizoaffective disorder will make another attempt within 2 years; within 10 years, $3 \%$ will have completed suicide, which represents about $60 \%$ of all patients with these diagnoses who complete suicide. When treating such high suicide risk patients, initiating or continuing treatment with clozapine must always be the initial consideration. Clozapine is the only treatment approved to reduce suicide risk in this (or any other) population. Its efficacy for this purpose is supported by the highest quality evidence, including a prospective, randomized controlled trial, InterSePT. A trial of clozapine should be strongly considered even for first-episode patients and those whose psychotic symptoms have responded to other antipsychotics, as amelioration of psychotic symptoms may be insufficient to reduce suicide risk. When recommending a trial of clozapine, thorough psychoeducation for the patient and his or her support system is necessary. This should include informing patients that indefinite treatment with clozapine may be needed because of the elevated risks of suicide and relapse to psychosis upon drug discontinuation. Discussion of the anticipated side effects and the providers' commitment to help minimize side effects are needed to make the trial of clozapine more attractive. If a trial of clozapine is unacceptable or impossible, alternative antipsychotic medications should be used and adherence monitored. A long-acting injectable atypical antipsychotic drug is preferable to oral medication, and certainly, to no antipsychotic treatment. The clinician should regularly inquire about suicidal thoughts and hopelessness and limit patients' access to means of self-harm. In addition, one should identify the symptoms, behaviors and social factors that contribute to each patient's suicide risk, and provide a combination of pharmacotherapy, psychosocial rehabilitation modalities, and psychotherapeutic interventions to address these factors. 


\section{Introduction}

$4.9 \%$ of individuals with schizophrenia will eventually complete suicide [1]. In 2010, 38,364 people died by suicide in the United States and approximately $7-12 \%$ of these are estimated to be suicides in individuals with schizophrenia, making suicide prevention in this population a major public health concern [2-4]. Those who complete suicide are disproportionately young adults, and suicide plays a major role in reduced longevity in schizophrenia. The mean age of those who commit suicide is just 33 years, and schizophrenia increases suicide risk 30-fold and 140-fold, respectively, for men and women under 35 years of age $[5,6]$. Many risk factors for suicide in patients with schizophrenia are those found in the general population, and include being young, male, white, never married, socially isolated, having prior suicide attempts, recent loss or rejection, and substance use disorder. Disease-specific risk factors include early phase of illness, with two-thirds of suicides occurring within 3 years of onset of illness, recent hospitalization, fear of mental deterioration, treatment non-adherence, and high pre-morbid functioning and awareness of impairments, which in conjunction may lead to demoralization and hopelessness $[7,8]$. Awareness of these risk factors can help clinicians identify those patients at high risk for suicide who should be offered targeted interventions to address this risk.

Clozapine stands alone as the only treatment that has been demonstrated to decrease suicide deaths in patients with schizophrenia. Consequently, it is the only medication with a United States Food and Drug Administration indication for reducing suicide risk in this population. In fact, clozapine is the only intervention that has consistently been shown to reduce mortality in patients with schizophrenia due to all-causes, including suicide. Tiihonen et al. demonstrated that clozapine's benefit over other antipsychotics in improving all-cause mortality is due, in large part but not exclusively, to its statistically significant superiority over other drugs in reducing suicide deaths. In this study, the adjusted risk for death from suicide on clozapine was just 0.34 , compared to 1.0 for perphenazine and 1.58 for quetiapine [9]. Remark- ably, it is estimated that if 10,000 patients with schizophrenia were treated with clozapine instead of standard treatment, 1,000 to 1,300 suicides would be prevented [10-12]. In addition, clozapine reduces suicide in high-risk patients with schizophrenia by approximately $80 \%$ compared to standard treatment $[13,12]$. Clozapine's superiority in diminishing suicidal behavior appears to be independent from its efficacy in treating positive, negative, or depressive symptoms, which suggests that perhaps suicidality should be viewed as a distinct dimension of the schizophrenia to be targeted individually [14]. Despite this clear benefit, clozapine is strikingly underutilized and efforts need to be made at the regulatory, educational, and quality improvement level to organize structures to increase its use [15]. In addition, we will briefly discuss management of the potentially serious side effects of clozapine (See Table 1 below), which may help increase its utilization, though it should be noted that clinicians may overestimate the burden of side effects and inconvenience that patients experience with clozapine [16]. The only other biological intervention recommended to address acute suicidality in schizophrenia is electroconvulsive therapy, though the evidence for this intervention in schizophrenia is inconsistent and may be best reserved in cases when used to augment clozapine [17-19].

Intuitively, it seems that another way to reduce suicide risk in schizophrenia should be to address modifiable risk factors; namely, treatment non-adherence, substance misuse, and depressive symptoms. However, there have been strikingly few studies investigating whether interventions targeting these risk factors actually change suicide-related outcomes in schizophrenia. In this article, we will review the current evidence base for treatments to address these modifiable suicide risk factors in schizophrenia, with a particular emphasis on biological interventions, but it should be noted that there is a clear and pressing need for further investigation to directly link these interventions to a reduction in suicide in schizophrenia. These interventions could then be incorporated into the algorithm for the approach to the suicidal patient with schizophrenia and schizoaffective disorder suggested by Meltzer et al. [20]. 


\begin{tabular}{lll}
\hline Table 1. Potential serious & side effects and management [26, 27] \\
\hline Side Effect & Management Options & $\begin{array}{l}\text { Re-Challenge } \\
\text { Potential } \\
\text { No }\end{array}$ \\
Agranulocytosis & $\begin{array}{l}\text { Discontinue Drug, Possible Hospital Admission, Granulocyte Colony } \\
\text { Stimulating Factors, Lithium }\end{array}$ & No \\
Myocarditis/ & Discontinue Drug, Possible Hospital Admission & \\
$\quad$ Cardiomyopathy & Prophylactic Laxatives, Diet modification & Yes \\
Ileus/Constipation & Metformin, Exercise/Diet, Topiramate & Yes \\
Weight Gain & Stimulants, Dosing Strategies, modafinil & Yes \\
Sedation & Lower dose, Divide dose, Valproic Acid, Lamotrigine & Yes \\
Seizures & Clonidine, Anticholinergics, Sugarless Rock Candy & Yes \\
Sialorrhea & Slow Titration, Compression Stockings, Increase Fluids, Fludrocortisone & Yes \\
Orthostatic Hypotension &
\end{tabular}

\section{Treatment}

\section{Pharmacologic treatment}

\section{Treatment of suicidal behavior in schizophrenia and for treatment resistant schizophrenia}

The gold standard study supporting the FDA indication for clozapine in suicidal patients is called the InterSept Study. This 2-year, prospective, international, multicenter, randomized study of 980 patients with schizophrenia at high risk for suicide showed a $26 \%$ reduction in the risk of suicide attempts in clozapine-treated patients compared to those using olanzapine. The NNT was just 12 for clozapine [21, Class I]. In addition to demonstrating this anti-suicide effect, this study also refuted the argument that this reduction is due to the increased provider contact needed for the administration of clozapine. It also showed that clozapine's reduction of suicide risk was seen in both treatment-resistant and treatment-responsive patients, which emphasized that the drug should be used to address suicidality in all patients with schizophrenia, not just the treatment resistant patients it is sometimes reserved for. Another study that controlled for clinical contact was published by Modestin et al. They used a mirror-image, retrospective design with hospitalized patients and found an $88 \%$ decrease in the suicides and a 12 -fold decrease in suicidal behaviors during the period of continuous clozapine treatment. In patients who discontinued clozapine, the rate of suicidal behavior increased to the baseline rate [22].

Further epidemiological studies and meta-analyses have added support to the evidence for clozapine in suicidal patients with schizophrenia. Walker et al undertook a large-scale retrospective epidemiologic study of 
Standard dosage

Contraindications

Main drug interactions

Main serious side effects and management options

special points

Cost/cost-effectiveness mortality and morbidity in 67,072 current and past clozapine users obtained from the US Clozaril National Registry. Deaths occurring in this cohort of patients were ascertained using the Social Security Administration Death Master Files and The National Death Index. An 83 \% reduction in death by suicide was observed in current clozapine users compared with past users [23]. John Hennen et al.'s random-effects meta-analysis indicated a substantially lower overall risk of suicidal behaviors with clozapine vs. other treatments (risk-ratio 3.3; $95 \%$ confidence interval [CI] $1.7-6.3 ; p=0.0001)$. For completed suicides, the risk ratio (RR) was 2.9 ([CI 1.5-5.7]; $p=0.002$ ) [24]. Kiviniemi et al published a nationwide, register-based, 5-year follow-up study of all patients presenting with first-onset of schizophrenia between 1998 and 2003. Use of clozapine reduced the likelihood of suicides (OR $0.29 ; P=0.002$ ) in patients with schizophrenia, supporting the conclusion that clozapine is effective in suicide reduction in all patients with schizophrenia: treatment resistant, treatment responsive, and even first-break patients [25•, Class 2]

300-900 mg per day in divided doses. Doses greater than $600 \mathrm{mg}$ increase risk of seizures. Gradual titration is recommended.

Agranulocytosis, chemotherapy, coma, ileus, leuokopenia, neutropenia, seizure disorder.

Smoking, concurrent use of strong CYP3A4 inducers is not recommended, Dosage reduction of clozapine is necessary during concurrent use of strong CYP1A2 inhibitors.

Separated from olanzapine at 3 months for suicide protection, so remember suicidal protective effects are delayed, plasma levels of clozapine ( $\geq 350 \mathrm{ng}$ / $\mathrm{ml}$ ) may be more effective, avoid abrupt discontinuation as may result in increased risk of rebound violent behavior, close monitoring of patients with Arab/African descent for WBC in 2,500-3,500 range, attempt to avoid antipsychotic polypharmacy, particularly with first generation antipsychotics as they may decrease efficacy of clozapine

Generic, Inexpensive

Antipsychotics: typical/FGA antipsychotics and atypical/SGA antipsychotics excluding clozapine

While clozapine is clearly superior and unique among antipsychotics in preventing suicides, for those patients unable or unwilling to take clozapine, antipsychotic treatment in general does seem to be useful in reducing suicide risk. Tiihonen et al. studied 2,230 adult patients with schizophrenia or schizoaffective disorder following initial admission and found that mortality was 12 times higher in patients not taking antipsychotics, and the risk of suicide in these patients was elevated 37.4 times [28]. Haukka et al. reported on 1,611 patients with a mean followup of 4.3 years and found that current antipsychotic use was associated with decreased mortality due to suicide (HR 0.52, $95 \%$ CI $0.34-0.81, p=$ 0.004) [29]. When comparing the data for the use of first generation 
antipsychotics (FGA) versus second generation antipsychotics (SGA) excluding clozapine, there is little high quality evidence that supports the use of FGA for suicide reduction [30]. There is some better general data on the use of SGA for the reduction of suicide, including a 5-year, retrospective, case control study that showed a decreased rate of suicide in patients using atypical antipsychotics (odds ratio $=3.54,95 \%$ confidence interval $=2.4$ to 5.3$)[31,32]$. However, direct, prospective high quality studies of individual SGA are lacking, and there does not seem to be any evidence that other agents possess the anti-suicide properties of clozapine.

Treatment of nonadherence in schizophrenia: long-acting injectables

Several review studies have directly linked nonadherence to an increase in suicide in schizophrenia. Leucht and Heres found that non-adherent patients were at four times greater risk of suicide than adherent patients (relative risk adjusted for age and gender 4.2, $95 \%$ CI 1.7-10.1) [33]. Llroca et al. found an even greater effect, as non-adherent patients were at seven times greater risk for suicide [34]. Herings and Erkens found that an increased suicide attempt rate was observed when comparing uninterrupted and interrupted drug use (20.0/1,000 person-years vs. 72.1/ 1,000 person years, respectively) [35]. The reasons for non-adherence are complex and many, but recent years have seen the development of additional long-acting injectable (LAI) formulations of second generation antipsychotics (SGA) to add to the previous armamentarium of first generation antipsychotic (FGA) LAI. The hope has been that these formulations can help with this issue of nonadherence, which could indirectly help with the issue of suicide in schizophrenia. However, recent meta-analyses of randomized controlled trials (RCTs) regarding LAI and adherence were disappointing. Kishimoto et al.'s review of 21 RCT studying a total of 4,950 patients showed that LAIs were similar to oral antipsychotics (OAP) for relapse prevention at the longest time point of relative risk $[\mathrm{RR}]=0.93,95 \%$ confidence interval $[\mathrm{CI}]: 0.80-1.08, P=$ 0.35 . Regarding nonadherence directly, pooled LAIs did not separate from OAPs (studies $=10, n=2018, \mathrm{RR}=0.77,95 \% \mathrm{CI}$ : 0.49-1.22, $P=0.22$. [36, Class I]. Kane et al. wrote a commentary that highlighted some of the methodological issues that may cause randomized controlled trials to underestimate the effectiveness of LAIs compared to OAPs. These include RCT patients being less ill and more adherent than real-world patients and current RCT procedures leading to an improvement in adherence. All of these issues would help to decrease any difference between OAP compared to LAI, as they would help the OAP adherence issue disproportionately [37]. In response to this, Kishimoto et al. ran a meta-analysis of 25 mirror-image studies from 28 countries that followed 5,940 patients with schizophrenia for $\geq 12$ months ( $\geq 6$ months each on oral antipsychotic and LAI treatment). The results showed strong superiority over oral antipsychotics in preventing hospitalization (16 studies, $N=$ 4,066; risk ratio $=0.43 ; 95 \% \mathrm{CI}, 0.35-0.53 ; P<0.001)$ and in decreasing the number of hospitalizations (15 studies, 6,342 person-years; rate ra- 
tio $=0.38 ; 95 \% \mathrm{CI}, 0.28-0.51 ; P<0.001)$ [38•, Class I]. While multiple limitations of mirror image studies exist, it is likely that this methodology is best at accurately assessing comparative efficacy of LAI in the naturalistic settings that most examiners practice. There is insufficient data to compare the different formulations of LAI regarding adherence, but Table 2 lists the newer and most frequently used LAI and summarizes some of the features that clinicians consider when choosing a particular LAI for their patients.

\section{Antidepressants}

There has been a surprising lack of quality studies investigating the impact of antidepressant use on depressive symptoms, suicidal ideation, and suicidal behavior in schizophrenia. [7, 39, 40]. In 2008, a Finnish registry-based prospective cohort study of 1,611 high-risk patients with schizophrenia and at least one prior suicide attempt found that current antidepressant use was not significantly associated with decreased risk of completed suicide (HR $0.66,95 \%$ CI $0.41-1.08, p=0.099$ ) [29]. However, in more detailed analysis, citalopram use was associated with significantly lower risk of death by suicide (HR 0.26; $95 \%$ CI 0.10-0.65) [29, Class II]. In the entire high-risk cohort, antidepressant use was actually associated with an increased likelihood of suicide attempts; however, this association was not found in patients who were concurrently receiving antipsychotic medications [29]. A second registry-based cohort study followed 2,588 patients for a mean of 4.2 years after initial hospitalization for schizophrenia, and found that current antidepressant use compared with no use was associated with significantly lower risk of completed suicide (HR 0.15, $95 \%$ CI 0.03-0.77) [41 • Class II]. However, a 2013 Swedish registry-based study of examined 84 matched case-control pairs of patients with schizophrenia or schizoaffective disorder, and found no significant association between completed suicide and lifetime history of either antidepressant use, or more specifically, selective serotonin reuptake

\begin{tabular}{|c|c|c|c|c|}
\hline LAI & $\begin{array}{l}\text { Frequency of } \\
\text { Dosing }\end{array}$ & $\begin{array}{l}\text { Need for Concominant } \\
\text { Oral Dosing (based in } \\
\text { part on clinical experience) }\end{array}$ & Special Points & Cost \\
\hline Abilify Maintenna & Monthly & 2 weeks & & Very Expensive \\
\hline Zyprexa Relprevv & $\begin{array}{l}\text { 2-4 weeks } \\
\text { (depending on } \\
\text { dosing desired) }\end{array}$ & None & $\begin{array}{l}\text { Post-Injection } \\
\text { Delirium/Sedation } \\
\text { Syndrome }\end{array}$ & Very Expensive \\
\hline Invega Sustenna & Monthly & 0-1 week & & Very Expensive \\
\hline Risperdal Consta & Every 2 weeks & 3-4 Weeks & & Expensive \\
\hline Haldol Decanoate & Every 4 weeks & 1-2 Weeks & $\begin{array}{l}\text { Conversion from Oral } \\
\text { to Decanoate is } \\
10-20 X \text { Oral dose }\end{array}$ & Inexpensive \\
\hline Prolixin Decanoate & Every 3-4 weeks & 1 Week & $\begin{array}{l}\text { Conversion from Oral } \\
\text { to Decanoate is } \\
1.2-1.4 X \text { Oral Dose }\end{array}$ & Inexpensive \\
\hline
\end{tabular}


inhibitor (SSRI) use [42, Class III]. A possible explanation for these conflicting findings could be that the smaller Swedish study was underpowered to detect this difference, as this study also failed to find a statistically significant association between clozapine use and suicide [42]. Additionally, as its authors note, the Swedish study only examined lifetime history of receiving an antidepressant prescription, and patients at higher risk of suicide from comorbid depression might have been more likely to have been prescribed antidepressants [42].

Only a handful of randomized placebo-controlled trials have investigated the question of whether antidepressants improve depressive symptoms or suicidal ideation in schizophrenia. In a 2002 meta-analysis, authors found that the overall poor quality of these studies left them unable to recommend or advise against the use of antidepressants in patients with schizophrenia. However, in 2009 and 2010, the largest to date randomized, double-blind, placebo-controlled trial of an SSRI in this population, demonstrated that citalopram improved depressive symptoms and suicidal ideation in this population [43, 44, Class I]. This study included 198 middle aged and older adult outpatients with a diagnosis of schizophrenia or schizoaffective disorder and sub-syndromal depressive symptoms [43,44]. The initial finding was that augmentation with citalopram (10-40 mg/day) led to statistically significant decrease in depressive symptoms and improvement in quality of life [43]. Subsequent analysis demonstrated that among patients who endorsed baseline suicidal ideation, those who received citalopram were significantly more likely than those who received placebo to deny suicidal ideation at study endpoint $(28.6 \%$ vs. $66.7 \%$; $P<0.05)$ [44]. Of note, there were no suicidal behaviors during the course of this trial, so actual impact of citalopram on completed suicide or attempts could not be determined.

Standard dosage 10 to $40 \mathrm{mg}$ per day.

Contraindications Concurrent monoamine oxidase inhibitor (MAOI) use. Citalopram causes dose-dependent QT prolongation, and is not recommended in patients already receiving medications that a prolong QT or inpatients with acute myocardial infarction, uncontrolled heart failure, bradycardia, hypokalemia, hypomagnesemia, or congenital long QT syndrome.

Main drug interactions There is a risk of serotonin syndrome when combined with other serotonergic medications.

Main side effects Drowsiness, insomnia, nausea, diarrhea, hyperhidrosis, decreased libido, erectile dysfunction

Cost/cost-effectiveness Inexpensive, as low as $\$ 4$ per month

\section{Treatment to reduce substance use}

$50 \%$ of patients with schizophrenia will develop comorbid substance use disorders, and the increased risk of suicide in these patients is likely related to the potential for substance misuse to increase impulsivity and violence, reduce overall functional status, promote social isolations and reduce treatment adherence [7, 11, 45-48]. For patients with comorbid schizophrenia 
and substance use disorder, the most effective treatment strategy appears to be an integrated approach that combines aggressive management of both disorders with a combination of pharmacotherapy and psychosocial interventions $[45,49]$.

There is evidence that choice of antipsychotic can impact substance use patterns in patients with schizophrenia, with clozapine having the best evidence supporting its use $[45,54,55]$. In a 3-year retrospective study of 58 current and former clozapine patients with co-occurring diagnoses of schizophrenia or schizoaffective disorder and substance use disorder, current clozapine use was associated with a marked reduction in substance use (85\% of those actively using substances at time of initiation of clozapine decreased their use, and $72 \%$ achieved abstinence) [56, Class II]. Additionally, an observational study of 66 patients with schizophrenia or schizoaffective disorder and comorbid alcohol use disorder, suggested that clozapine patients were more than twice as likely to have achieved substance use remission than patients taking typical antipsychotics [57, Class II]. In 2003, another retrospective study examined substance use patterns in 68 patients with schizophrenia or schizoaffective disorder and alcohol or marijuana abuse, who were prescribed either risperidone or clozapine, and found that patients taking clozapine were significantly more likely to abstain from substances [58, Class II]. In a 2006 prospective study of 75 patients with diagnoses of substance use disorder in early remission and either schizophrenia or schizoaffective disorder, clozapine users were significantly less likely to relapse than patients prescribed other antipsychotics [59, Class II].

There have been few studies investigating the use of medications to address specific substance use in patients with schizophrenia. Two small randomized placebo-controlled studies demonstrated that naltrexone (compared to placebo) reduced alcohol consumption in patients with comorbid primary psychotic disorder and alcohol use disorder [60-62, Class II]. There is very little evidence on methadone replacement and buprenorphine use in patients with schizophrenia and opiate use disorders: one small case series suggests potential benefit from methadone, and one uncontrolled study suggested that patients with comorbid schizophrenia and opiate use disorder benefitted less from buprenorphine than patients with other psychiatric disorders [63, 64].

Standard dosage

Contraindications

Main drug interactions

Main side effects
$50 \mathrm{mg}$ once daily with food; some patients may require up to $100 \mathrm{mg}$. Alternatively, patients who demonstrate the ability abstain from alcohol use in an outpatient setting may receive $380 \mathrm{mg}$ IM every 4 weeks.

Absolute contraindications are hypersensitivity, concurrent use of opiate analgesics and acute opiate withdrawal.

Opiate agonists, mixed opiate agonists/antagonists, nilotinib (tyrosine kinase inhibitor). May have additive hepatotoxic effect when combined with disulfuram.

Dizziness or syncope, anxiety, insomnia, headache, nausea, injection site reactions (with injectable form), liver toxicity, may increase suicidal thoughts. 
Special points Only start naltrexone if there is no reasonable possibility within of shortacting opiate use in the past 7-10 days or long-acting opiate use in the past 2 weeks. If there is any suspicion of opiate use, naloxone challenge should be tried prior to naltrexone initiation.

Cost/Cost-effectiveness Oral: $\$ 50-150$ per month, injectable Vivitrol $\$ 1,050$ per month

\section{Interventional procedures}

Electroconvulsive therapy (ECT) is recommended for acutely suicidal patients with schizophrenia, primarily because of its potential to produce rapid improvement in symptoms compared to pharmacologic treatments [17, 65-67]. However, the empirical evidence supporting the use of ECT for reducing suicide risk in schizophrenia is scant and inconsistent [17]. In the 1940s, two case-control studies of patients with affective psychosis suggested that treatment with ECT was associated with a decreased risk of death by suicide [68, 69, Class III]. These findings were not reproduced in two subsequent observational studies in the 1950s, which found no significant association between ECT and suicide risk in patients with diagnoses of involutional psychosis or manic-depressive illness [70, 71]. A 1979 observational study of 74 patients with schizoaffective disorder found significantly decreased overall mortality and death by suicide in those treated with ECT [72]. In contrast, a 1986 an observational study of 1,494 psychiatric inpatients failed to demonstrate any significant decrease in suicide in patients treated with ECT, and this finding remained in subsequent analysis of only patients with schizophrenia [73]. Most recently in 2007, a Danish national registry-based cohort study examined mortality and suicide outcomes in 783 deceased psychiatric inpatients who had received ECT and 5,781 inpatients who had not [74]. Patients who had received ECT had a lower mortality rate from natural causes, but a marginally significant higher suicide rate (RR 1.20, $95 \%$ CI 0.99-1.47), which was more pronounced within 7 days of last ECT treatment (RR. 4.82, $95 \%$ CI 2.12-10.95) [74]. However, authors note that risk was likely due to selection bias, as they were unable to control for pre-existing suicide risk factors, and patients at higher risk of suicide may have received ECT at a higher rate [75]. Thus, while ECT may be useful in preventing imminent self-harm in some patients with schizophrenia, its potential to modify ongoing suicide risk in this population remains unclear.

Standard procedure A series of treatments in which unilateral or bilateral electrodes are used to deliver an electrical stimulus sufficient to cause a seizure, performed under anesthesia and by a trained specialist.

Contraindications No absolute contraindications. Certain medical conditions such as structural brain disease, increased intracranial pressure, pulmonary disease and cardiac disease require careful analysis of risks versus benefits and close monitoring.

Complications Cognitive impairment, anterograde and retrograde amnesia, headache, musculoskeletal pain. Less common but serious complications include ar- 
Cost/cost-effectiveness Expensive, cost varies depending on medical center.

\section{Psychotherapy and psychosocial interventions}

There are remarkably few studies examining the impact of specific psychotherapeutic and psychosocial interventions on suicide risk in patients with schizophrenia [47]. A 2007, randomized controlled trial of cognitive behavioral therapy (CBT) in 90 outpatients with schizophrenia, found that cognitive behavioral therapy led to significant reduction in suicidal ideation compared to "befriending," and that this improvement continued for at least 9 months after completion of therapy intervention [76,Class II]. A 2002 randomized trial comparing CBT to treatment as usual in 422 patients with schizophrenia found that CBT was superior in ameliorating depressive symptoms, with a number needed to treat of nine [77,Class I]. A methodologically rigorous 2008 meta-analysis of CBT for schizophrenia suggested that CBT produces a modest yet significant improvement in mood symptoms (weighted effect size 0.363, $95 \%$ CI 0.079-0.647), but authors found significant heterogeneity in the effect sizes among the 15 different trials that assessed mood) [78, Class II]. This meta-analysis did not find any significant impact of CBT on hopelessness, which was measured in only four trials [78].

Additionally, there is some evidence that psychosocial interventions such as assertive community treatment, reality-orientated psychotherapy, family psycho-education, and the use of token economy can improve treatment adherence in patients with schizophrenia [79-81]. Evidence-based psychosocial treatments to reduce substance use in patients with schizophrenia include group therapy that combines CBT and motivational enhancement techniques, contingency management, and residential treatment when outpatient management is insufficient [45, 50-53].

\section{Compliance with Ethics Guidelines}

\section{Conflict of Interest}

Dr. Herbert Meltzer has received honoraria for consulting, or research contracts within the last 5 years from Alkermes, Astellas, EnVivo, DaiNippon Sumitomo, Forest, Merck, Naurex, Novartis, Otsuka, Sunovion Pharmaceuticals Inc., SureGene, Takeda, and TEVA. Dr. Meltzer was the Principal Investigator of the InterSePT study, funded by Novartis and has prepared an educational program regarding suicide and clozapine sponsored by Teva. He has served as a board member or received payment to lecture at Acadia, Asenapine, BioLine Rx, Boehringer Ingelheim Pharma GmbH \&Co. KG, Janssen, Sunovion, SureGene, and Teva, Additionally, Dr. Meltzer owns stock or stock options in Arcadia, SureGene and GlaxoSmithKline.

Dr. Gaurava Agarwal has received salary support for clinical research from Takeda, Naurex, Otsuka, and Sunovion Pharmaceuticals.

Dr. Megan Pirigyi has no disclosures to report.

Human and Animal Rights and Informed Consent

This article does not contain any studies with human or animal subjects performed by any of the authors. 
Papers of particular interest, published recently, have been highlighted as:

- Of importance

1. Palmer BA, Pankratz VS, Bostwick JM. The lifetime risk of suicide in schizophrenia: a reexamination. Arch Gen Psychiatry. 2005;62:247-53.

2. Qin P, Nordentoft M. Suicide risk in relation to psychiatric hospitalization: evidence based on longitudinal registers. Arch Gen Psychiatry. 2005;62:427-32.

3. Allebeck P. Schizophrenia: a life-shortening disease. Schizophr Bull. 1989;15:81-9.

4. Caldwell CB, Gottesman II. Schizophrenics kill themselves too: a review of risk factors for suicide. Schizophr Bull. 1990;16:571-89.

5. Qin P. The impact of psychiatric illness on suicide: differences by diagnosis of disorders and by sex and age of subjects. J Psychiatr Res. 2011;45:1445-52.

6. Roy A. Depression, attempted suicide, and suicide in patients with chronic schizophrenia. Psychiatr Clin N Am. 1986;9:193-206.

7. Hawton K, Sutton L, Haw C, Sinclair J, Deeks JJ. Schizophrenia and suicide: systematic review of risk factors. Br J Psychiatry. 2005;187:9-20.

8. Alaraisanen A, Miettunen J, Rasanen P, Fenton W, Koivumaa-Honkanen HT, Isohanni M. Suicide rate in schizophrenia in the Northern Finland 1966 Birth Cohort. Soc Psychiatry Psychiatr Epidemiol. 2009;44:1107-10.

9. Tiihonen J, Lonnqvist J, Wahlbeck K, et al. 11-year follow-up of mortality in patients with schizophrenia: a population-based cohort study (FIN11 study). Lancet. 2009;374:620-7.

10. Wagstaff A, Perry C. Clozapine: in prevention of suicide in patients with schizophrenia or schizoaffective disorder. CNS Drugs. 2003;17:27380. discussion 81-3.

11. Roy A, Pompili M. Management of schizophrenia with suicide risk. Psychiatr Clin N Am. 2009;32:86383.

12. Meltzer HY, Okayli G. Reduction of suicidality during clozapine treatment of neuroleptic-resistant schizophrenia: impact on risk-benefit assessment. Am J Psychiatr. 1995;152:183-90.

13. Reid WH, Mason M, Hogan T. Suicide prevention effects associated with clozapine therapy in schizophrenia and schizoaffective disorder. Psychiatr Serv. 1998;49:1029-33.

14. Meltzer HY. Clozapine: balancing safety with superior antipsychotic efficacy. Clin Schizophr Relat Psychoses. 2012;6:134-44.

15. Horvitz-Lennon M, Donohue JM, Domino ME, Normand SL. Improving quality and diffusing best practices: the case of schizophrenia. Health Aff (Millwood). 2009;28:701-12.

16. Hodge K, Jespersen S. Side-effects and treatment with clozapine: a comparison between the views of consumers and their clinicians. Int J Ment Health Nurs. 2008;17:2-8.

17. Pompili M, Lester D, Dominici G, et al. Indications for electroconvulsive treatment in schizophrenia: a systematic review. Schizophr Res. 2013;146:1-9.

18. Flamarique I, Castro-Fornieles J, Garrido JM, et al. Electroconvulsive therapy and clozapine in adolescents with schizophrenia spectrum disorders: is it a safe and effective combination? J Clin Psychopharmacol. 2012;32:756-66.

19. Kupchik M, Spivak B, Mester R, et al. Combined electroconvulsive-clozapine therapy. Clin Neuropharmacol. 2000;23:14-6.

20. Meltzer HY, Conley RR, De Leo D, Green AI, Kane JM, Knesevich MA, et al. Intervention strategies for suicidality. J Clin Psychiatry. 2003;6:1-16.

21. Meltzer HY, Alphs L, Green AI, et al. Clozapine treatment for suicidality in schizophrenia: International Suicide Prevention Trial (InterSePT). Arch Gen Psychiatry. 2003;60:82-91.

22. Modestin J, Dal Pian D, Agarwalla P. Clozapine diminishes suicidal behavior: a retrospective evaluation of clinical records. J Clin Psychiatry. 2005;66:534-8.

23. Walker AM, Lanza LL, Arellano F, Rothman KJ. Mortality in current and former users of clozapine. Epidemiology. 1997;8:671-7.

24. Hennen J, Baldessarini RJ. Suicidal risk during treatment with clozapine: a meta-analysis. Schizophr Res. 2005;73:139-45.

25. Kiviniemi M, Suvisaari J, Koivumaa-Honkanen $\mathrm{H}$, Hakkinen $\mathrm{U}$, Isohanni $\mathrm{M}$, Hakko $\mathrm{H}$. Antipsychotics and mortality in first-onset schizophrenia: prospective Finnish register study with 5-year follow-up. Schizophr Res. $2013 ; 150: 274-80$.

[Class 2] This article supports clozapine's ability to reduce suicide risk in first onset schizophrenia, supporting the contention that clozapine should not be reserved for only patients that are treatment resistant in cases where suicide risk is high.

26. Nielsen J, Correll CU, Manu P, Kane JM. Termination of clozapine treatment due to medical reasons: when is it warranted and how can it be avoided? J Clin Psychiatry. 2013;74:603-13. quiz 13. 
27. Young CR, Bowers Jr MB, Mazure CM. Management of the adverse effects of clozapine. Schizophr Bull. 1998;24:381-90.

28. Tiihonen J, Wahlbeck K, Lonnqvist J, et al. Effectiveness of antipsychotic treatments in a nationwide cohort of patients in community care after first hospitalisation due to schizophrenia and schizoaffective disorder: observational follow-up study. BMJ. 2006;333:224.

29. Haukka J, Tiihonen J, Harkanen T, Lonnqvist J. Association between medication and risk of suicide, attempted suicide and death in nationwide cohort of suicidal patients with schizophrenia.

Pharmacoepidemiol Drug Saf. 2008;17:686-96.

30. Palmer DD, Henter ID, Wyatt RJ. Do antipsychotic medications decrease the risk of suicide in patients with schizophrenia? J Clin Psychiatry. 1999;60 Suppl 2:100-3. discussion 11-6.

31. Barak Y, Mirecki I, Knobler HY, Natan Z, Aizenberg D. Suicidality and second generation antipsychotics in schizophrenia patients: a case-controlled retrospective study during a 5-year period. Psychopharmacology (Berl). 2004;175:215-9.

32. Keck Jr PE, Strakowski SM, McElroy SL. The efficacy of atypical antipsychotics in the treatment of depressive symptoms, hostility, and suicidality in patients with schizophrenia. J Clin Psychiatry. 2000;61 Suppl 3:4-9.

33. Leucht S, Heres S. Epidemiology, clinical consequences, and psychosocial treatment of nonadherence in schizophrenia. J Clin Psychiatry. 2006;67 Suppl 5:3-8.

34. Llorca PM. Partial compliance in schizophrenia and the impact on patient outcomes. Psychiatry Res. 2008;161:235-47.

35. Herings RM, Erkens JA. Increased suicide attempt rate among patients interrupting use of atypical antipsychotics. Pharmacoepidemiol Drug Saf. 2003;12:423-4.

36. Kishimoto T, Robenzadeh A, Leucht C, et al. Longacting injectable vs oral antipsychotics for relapse prevention in schizophrenia: a meta-analysis of randomized trials. Schizophr Bull. 2014;40(1):192-213

37. Kane JM, Kishimoto T, Correll CU. Assessing the comparative effectiveness of long-acting injectable vs. oral antipsychotic medications in the prevention of relapse provides a case study in comparative effectiveness research in psychiatry. J Clin Epidemiol. 2013;66:S37-41.

38. Kishimoto T, Nitta M, Borenstein M, Kane JM, Correll CU. Long-acting injectable versus oral antipsychotics in schizophrenia: a systematic review and metaanalysis of mirror-image studies. J Clin Psychiatry. 2013;74:957-65.

[Class 1] This article lends support to the idea that longacting injectable formulations of antipsychotics are helpful in increasing adherence to medications.
39. Whitehead C, Moss S, Cardno A, Lewis G. Antidepressants for people with both schizophrenia and depression. Cochrane Database Syst Rev. 2002. doi:10.1002/14651858.CD002305.

40. Siris SG, Addington D, Azorin JM, Falloon IR, Gerlach J, Hirsch SR. Depression in schizophrenia: recognition and management in the USA. Schizophr Res. 2001;47:185-97.

41.• Tiihonen J, Suokas JT, Suvisaari JM, Haukka J, Korhonen P. Polypharmacy with antipsychotics, antidepressants, or benzodiazepines and mortality in schizophrenia. Arch Gen Psychiatry. 2012;69:47683.

[Class 1] This article reviews the use of multiple of classes of medications in reducing mortality, including suicidality, in patients with schizophrenia.

42. Reutfors J, Bahmanyar S, Jonsson EG, et al. Medication and suicide risk in schizophrenia: a nested casecontrol study. Schizophr Res. 2013;150:416-20.

43. Zisook S, Kasckow JW, Golshan S, et al. Citalopram augmentation for subsyndromal symptoms of depression in middle-aged and older outpatients with schizophrenia and schizoaffective disorder: a randomized controlled trial. J Clin Psychiatry. 2009;70:562-71.

44. Zisook S, Kasckow JW, Lanouette NM, et al. Augmentation with citalopram for suicidal ideation in middle-aged and older outpatients with schizophrenia and schizoaffective disorder who have subthreshold depressive symptoms: a randomized controlled trial. J Clin Psychiatry. 2010;71:915-22.

45. Lybrand J, Caroff S. Management of schizophrenia with substance use disorders. Psychiatr Clin. 2009;32:821-33.

46. Kasckow J, Felmet K, Zisook S. Managing suicide risk in patients with schizophrenia. CNS Drugs. 2011;25:129-43.

47. Carlborg A, Winnerback K, Jonsson EG, Jokinen J, Nordstrom P. Suicide in schizophrenia. Expert Rev Neurother. 2010;10:1153-64.

48. Baeza I, Flamarique I, Garrido JM, et al. Clinical experience using electroconvulsive therapy in adolescents with schizophrenia spectrum disorders. J Child Adolesc Psychopharmacol. 2010;20:205-9.

49. Drake RE, Essock SM, Shaner A, et al. Implementing dual diagnosis services for clients with severe mental illness. Psychiatr Serv. 2001;52:469-76.

50. Bellack AS, Bennett ME, Gearon JS, Brown CH, Yang Y. A randomized clinical trial of a new behavioral treatment for drug abuse in people with severe and persistent mental illness. Arch Gen Psychiatry. 2006;63:426-32.

51. Haddock G, Barrowclough C, Tarrier N, et al. Cognitive-behavioural therapy and motivational intervention for schizophrenia and substance misuse. 18month outcomes of a randomised controlled trial. $\mathrm{Br}$ J Psychiatry. 2003;183:418-26. 
52. Brunette MF, Drake RE, Woods M, Hartnett T. A comparison of long-term and short-term residential treatment programs for dual diagnosis patients. Psychiatr Serv. 2001;52:526-8.

53. Barrowclough $\mathrm{C}$, Haddock G, Wykes $\mathrm{T}$, et al. Integrated motivational interviewing and cognitive behavioural therapy for people with psychosis and comorbid substance misuse: randomised controlled trial. BMJ. 2010;341:c6325.

54. Kelly TM, Daley DC, Douaihy AB. Treatment of substance abusing patients with comorbid psychiatric disorders. Addict Behav. 2012;37:11-24.

55. San L, Arranz B, Martinez-Raga J. Antipsychotic drug treatment of schizophrenic patients with substance abuse disorders. Eur Addict Res. 2007;13:230-43.

56. Zimmet SV, Strous RD, Burgess ES, Kohnstamm S, Green AI. Effects of clozapine on substance use in patients with schizophrenia and schizoaffective disorder: a retrospective survey. J Clin Psychopharmacol. 2000;20:94-8.

57. Drake RE, Xie H, McHugo GJ, Green AI. The effects of clozapine on alcohol and drug use disorders among patients with schizophrenia. Schizophr Bull. 2000;26:441-9.

58. Green AI, Burgess ES, Dawson R, Zimmet SV, Strous RD. Alcohol and cannabis use in schizophrenia: effects of clozapine vs. risperidone. Schizophr Res. 2003;60:81-5.

59. Brunette MF, Drake RE, Xie H, McHugo GJ, Green AI. Clozapine use and relapses of substance use disorder among patients with co-occurring schizophrenia and substance use disorders. Schizophr Bull. 2006;32:637-43.

60. Green AI, Noordsy DL, Brunette MF, O'Keefe C. Substance abuse and schizophrenia: pharmacotherapeutic intervention. J Subst Abus Treat. 2008;34:61-71.

61. Petrakis IL, O'Malley S, Rounsaville B, et al. Naltrexone augmentation of neuroleptic treatment in alcohol abusing patients with schizophrenia.

Psychopharmacology. 2004;172:291-7.

62. Petrakis IL, Nich C, Ralevski E. Psychotic spectrum disorders and alcohol abuse: a review of pharmacotherapeutic strategies and a report on the effectiveness of naltrexone and disulfiram. Schizophr Bull. 2006;32:644-54.

63. Miotto P, Preti A, Frezza M. Heroin and schizophrenia: subjective responses to abused drugs in dually diagnosed patients. J Clin Psychopharmacol. 2001;21:111-3.

64. Gerra G, Leonardi C, D'Amore A, et al. Buprenorphine treatment outcome in dually diagnosed heroin dependent patients: a retrospective study. Prog Neuro-Psychopharmacol Biol Psychiatry. 2006;30:265-72.
65. Greenblatt M. Efficacy of ECT in affective and schizophrenic illness. Am J Psychiatry. 1977;134:1001-5.

66. Small JG, Milstein V, Klapper M, Kellams JJ, Small IF. ECT combined with neuroleptics in the treatment of schizophrenia. Psychopharmacol Bull. 1982;18:34-5.

67. Abraham KR, Kulhara P. The efficacy of electroconvulsive therapy in the treatment of schizophrenia. A comparative study. Br J Psychiatry. 1987;151:152-5.

68. Ziskind E, Somerfeld-Ziskind E, Ziskind L. Metrazol and electric convulsive therapy of the affective psychoses. Arch Neurol Psychiatry. 1945;53:212-7.

69. Huston PE, Locher LM. Involutional psychosis; course when untreated and when treated with electric shock. Arch Neurol Psychiatry. 1948;59:385-94.

70. Bond ED. Results of treatment in psychoses with a control series. II. Involutional psychotic reaction. Am J Psychiatry. 1954;110:881-3.

71. Bond ED, Morris Jr HH. Results of treatment in psychoses-with a control series. III. Manic-depressive reactions. Am J Psychiatry. 1954;110:883-5.

72. Tsuang MT, Dempsey GM, Fleming JA. Can ECT prevent premature death and suicide in 'schizoaffective' patients? J Affect Disord. 1979;1:167-71.

73. Milstein V, Small JG, Small IF, Green GE. Does electroconvulsive therapy prevent suicide? Convuls Ther. 1986;2:3-6.

74. Munk-Olsen T, Laursen TM, Videbech P, Mortensen $\mathrm{PB}$, Rosenberg R. All-cause mortality among recipients of electroconvulsive therapy: register-based cohort study. Br J Psychiatry. 2007;190:435-9.

75. Laursen TM, Munk-Olsen T, Nordentoft M, Bo Mortensen P. A comparison of selected risk factors for unipolar depressive disorder, bipolar affective disorder, schizoaffective disorder, and schizophrenia from a danish population-based cohort. J Clin Psychiatry. 2007;68:1673-81.

76. Bateman AW, Ryle A, Fonagy P, Kerr IB. Psychotherapy for borderline personality disorder: mentalization based therapy and cognitive analytic therapy compared. Int Rev Psychiatry. 2007;19:5162.

77. Turkington D, Kingdon D, Turner T. Insight into schizophrenia research G. Effectiveness of a brief cognitive-behavioural therapy intervention in the treatment of schizophrenia. Br J Psychiatry. 2002;180:523-7.

78. Wykes T, Steel C, Everitt B, Tarrier N. Cognitive behavior therapy for schizophrenia: effect sizes, clinical models, and methodological rigor. Schizophr Bull. 2008;34:523-37.

79. Dixon LB, Dickerson F, Bellack AS, et al. The 2009 schizophrenia PORT psychosocial treatment recommendations and summary statements. Schizophr Bull. 2010;36:48-70. 
80. Bush CT, Langford MW, Rosen P, Gott W. Operation outreach: intensive case management for severely psychiatrically disabled adults. Hosp Community Psychiatry. 1990;41:647-9. discussion 9-51.
81. Falloon IR, Boyd JL, McGill CW, et al. Family management in the prevention of morbidity of schizophrenia. Clinical outcome of a two-year longitudinal study. Arch Gen Psychiatry. 1985;42:887-96. 